\title{
Solar Radiation, Perelman Entropy Mapping, DNA, Viruses etc.
}

\author{
Kai W. Wong1, Peter C. W. Fung², Wan K. Chow ${ }^{3}$ \\ ${ }^{1}$ Department of Physics and Astronomy, University of Kansas, Lawrence, USA \\ ${ }^{2}$ Department of Physics, Department of Medicine, and Centre on Behavioral Health, University of Hong Kong, Hong Kong, China \\ ${ }^{3}$ Department of Building Services Engineering, The Hong Kong Polytechnic University, Hong Kong, China \\ Email:kww88ng@gmail.com
}

How to cite this paper: Wong, K.W., Fung, P.C.W. and Chow, W.K. (2020) Solar Radiation, Perelman Entropy Mapping, DNA, Viruses etc. Open Journal of Biophysics, 10, 54-58.

https://doi.org/10.4236/ojbiphy.2020.102005

Received: February 10, 2020

Accepted: March 13, 2020

Published: March 16, 2020

Copyright (C) 2020 by author(s) and Scientific Research Publishing Inc. This work is licensed under the Creative Commons Attribution International License (CC BY 4.0).

http://creativecommons.org/licenses/by/4.0/

\begin{abstract}
A short note based on the homogeneous 5D space-time topological mappings is extended to cover DNAs of viruses and how the body's immune system can be enhanced to recognize and remove it.

\section{Keywords}

5D Homogeneous Space-Time and Perelman Mappings, Nitrogenous Bases, DNA, Viruses, Coronavirus, Immune System
\end{abstract}

Recently for the first time a detailed picture of the solar surface was observed via frequency patent analysis. It was found that its intensity patent resembles that of the Sunflower (see Figure 1).

While it is not expected, it actually represents the geometric patent of the result from Perelman Quantum entropy mapping that gives us the Poincare sphere, via the breaking of the Maxwell monopole potential fields. We refer our readers to the published articles [1] [2] [3] [4]. The results can be summarized from the breaking of the 5D quantum homogeneous space-time manifold vector field potentials as given by the direct product of the 4 Maxwell E-M potentials with the Maxwell monopole potentials into the non-homogeneous $\{\mathrm{SU}(2)+$ $\mathrm{SU}(3)\} \times \mathrm{xL}$ obtained via the dimension reduction projections Po and P1 as discussed in [3] [4]. It is through such projections and the conserving of gauge invariance as required by the coupling between the vector and e-trino [1] spinor solutions that matter as represented by hadrons and leptons are produced. And through the requirement of maintaining charge neutrality as given by the $5 \mathrm{D}$ homogeneous space-time, elements and complex molecules are formed in place of a homogeneous matter distribution. In fact the lowest two Poincare quantum 


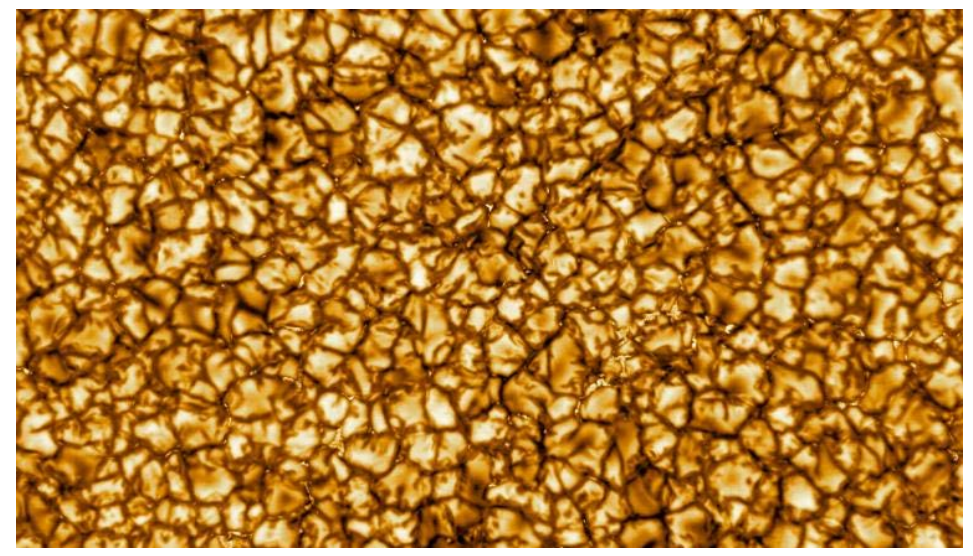

Figure 1. Solar surface (image source: https://www.nationalgeographic.com/science/2020/02/sun-still-burningmystery-may-be-about-to-change-solar-orbiter-launch/.

spheres formed through Perelman mappings [2] and through the application of Gell-Mann standard model [5] are that of the Carbon 12 nucleus as geometrically represented by the hard sphere dense packing [6], while the next level is that given by the Bucky Ball surface of protons and neutrons. It is then easy to see that the fundamental patents on the Poincare spherical surface are simply that of the hexagon and the pentagon (see Figure 2 and Figure 3).

The basic matter creation then splits the energy domain of the monopole fields, resulting in leaving the energy portion that is less than the rest mass of the electron still could be preserving the homogeneous 5D symmetry. It is only through the further spherical space geometrical symmetry breaking into $2 \mathrm{D} \times$ $1 \mathrm{D}$, and the maintenance of gauge invariance that we are led to the formation of the four basic nitrogenous bases [7], essential to the creation of DNAs obtained from the random stacking of the bases and thereby creates the life forms. In order to achieve that $2 \mathrm{D}$ space symmetry for the nitrogenous bases, we establish the normal by adding two neutrons on opposite equilateral triangles in $\mathrm{C} 12$ shell, creating C14; thus all live forms contain C14. While for the Buckyball shell, neutrons can be added to centers of the pentagons, making a sphere with spikes. In the bio micro-scale, these spikes contain the RNAs. The identification of each life form is then given by the frequency spectra, which in turn is completely determined by the quantum phase matching tunneling of the Diagonal-Long-Range-Order, DLRO, monopole fields strength, formed from two opposite charge and momenta massless spinous, that is the etrino and anti-etrino, with a total angular momentum 1, similar to the photon and therefore depends explicitly on the length of each section of random stacking of the nitrogenous bases and the matching quantum tunneling phases in its DNAs. The life form's genome number is thus given by $2 \times 7 ! \times 3=30240$, where the factor 3 comes from the photon angular momentum, and the factor 7 is because the end caps must be only given by the end nitrogenous base Thymine, with only a single transmission face. It is via the DLRO boson state that induces the 


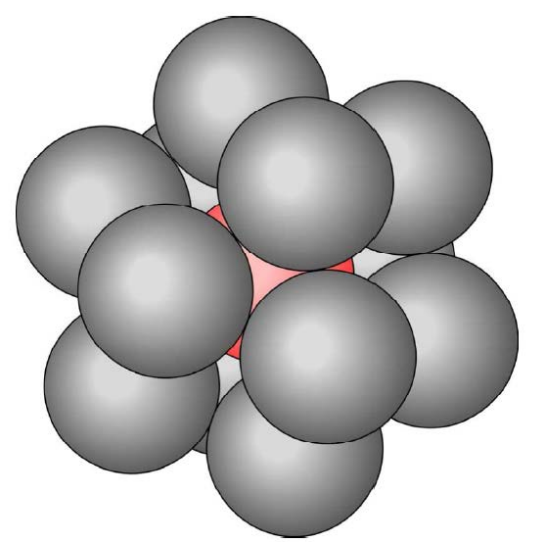

Figure 2. C12 hard sphere dense packing model (Wong et al. 2014, ref. [1]).

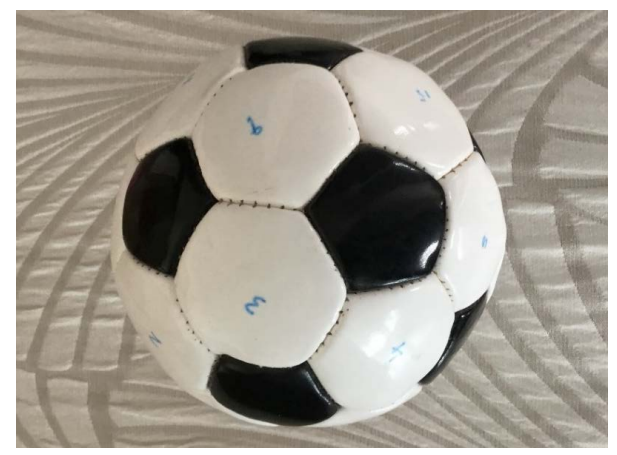

Figure 3. The 20 hexagons are in white, while the 12 isolated pentagons are in black.

Off-Diagonal-Long-Range-Order, ODLRO in the chemical valence band states provided by the electronic $\mathrm{p}$ waves in the carbon of the bio-matter that then leads to the natural growth of biological cells, body tissues, organs and structure [8] [9]. Thus each life possesses a unique set of cell structures identified by its DNAs spectrum. Any non-compliance should be detected by the body's natural immune systems and eliminated from self reproduction. But due to the infinite choices in the varying spectra possible, certain closed foreign DNAs are frequently missed by the immune system detection. It is this problem that leads to the invasion of foreign DNAs that can be separated into non-cell structure viruses, such as the common flus, SAR and the recent coronavirus, etc. provided the effective body's thermal temperature $\mathrm{T}<\mathrm{Tc}$ of the virus critical temperature for growth, thus allowing it to multiply within the body leading usually to destruction of the lung cells and the prevention of the body's respiratory natural oxidation process in the removal of the dead cells within the body. Meaning these viruses always disrupt the body's respiratory function, because from PV/T constrain as $\mathrm{PV}$ decrease, where $\mathrm{P}$ and $\mathrm{V}$ are air pressure and volume of the lung respectively, so will $\mathrm{T}$, enhancing the virus reproduction. Thus the coronavirus might behave like the common flu and is perhaps seasonal. While other foreign DNAs left within the body coupled to the cell and tissue growth would result in 
cancerous growth of the body. Because of these, there are numerous potential harms that can cause the death of life without making the immune system recognizing and remove these foreign DNAs. Understanding the fundamental physics could guide us to develop proper cure and preventions. Take as an example, small pox vaccine was derived from dead tissues formed from small pox. The current coronavirus, is supposed to be originated from the bat bitting of the snake that created this virus and passes it onward to human. Normally in the wild, that could be a frequent occurrence. But the bitten snake dies and the virus with it disappeared. Yet in a market, both snakes and other animals are placed in contact, hence leading to the virus being spread and multiplied. If true, it means the COVID-19 can start anywhere, even without human transmission. This coronavirus is unique in that it composes the DNA spectra from the warm blooded bat to that of the cold blooded snake. Cold blooded animal is similar to plants, as such part of its DNA spectra must contain that is the visible portion of spectrum as provided by the solar photo spectrum. Meaning this coronavirus DNA is unlike the SAR and EBOLA, which have DNA spectra covering only those of warm blooded mammals, must contain such optical photon frequency portion also. Hence photo UV irradiation will not kill the coronavirus. The human body's immune system would be activated if it recognizes it as being foreign. Since human is warm-blooded, it is more likely for its immune system to detect this virus and respond to eliminate it, unless that human body immune system is weak. Perhaps this explains why the coronavirus so far indicated is far less deadly than SAR or EBOLA, and it does not seem to be deadly for the young as their immune system and lung are still developing? To see how we can normally strengthen our immune system to beat back the Coronavirus, we can fall back on what we know. If we consume raw honey which is sugar obtained by the bees pollination of different flowers, it could stimulate the body's immune system to recognize those plant's DNA that possesses spectrum in the visible and UV range, which might even cover that of the coronavirus? In fact by analyzing a virus DNA spectrum we can create medications by matching certain known immune enhancement medication or food that will induce the human body immune system to recognize and remove it.

\section{Acknowledgements}

We thank Ms. Winnie So for her great help in preparing the manuscript.

\section{Conflicts of Interest}

The authors declare no conflicts of interest regarding the publication of this paper.

\section{References}

[1] Wong, K.W., Dreschhoff, G.A.M. and Jungner, H. (2014) The Five Dimension Space-Time Universe-A Creation and Grand Unified Field Theory Model. Scien- 
tific Research Publishing, Wuhan.

[2] Wong, K.W., Dreschhoff, G., Jungner, H., Fung, P.C.W. and Chow, W.K., (2018) The Magnetic Monopole in 5D Homogeneous Space-Time. Physics Essays, 31, 493-495. https://doi.org/10.4006/0836-1398-31.4.493

[3] Perelman, G. (2002) The Entropy Formula for the Ricci Flow and Its Geometric Application. arXiv.math.DG/0211159.

Perelman, G. (2003) Ricci Flow with Surgery on Three-Manifolds.

arXiv.mathDG/0303109.

[4] Wong, K.W., Fung, P.C.W. and Chow, W.K., (2019) A Quantum Representation of the Homogeneous 5D Manifold and the Perelman Mappings of 5D onto Non-Homogeneous Lorentz 4D Manifolds. Journal of Modern Physics, 10, 557-575. https://doi.org/10.4236/jmp.2019.105039

[5] Gell-Mann, M. (1964) Nonleptonic Weak Decays and the Eightfold Way. Physical Review Letters, 12, 155-156. https://doi.org/10.1103/PhysRevLett.12.155

[6] Wong, K.W. (1964) Application of Nonlocal Field Operators to a System of Hard Sphere Bose Gas. Journal of Mathematical Physics, 5, 637-642.

https://doi.org/10.1063/1.1704157

[7] Wong, K.W., Fung, P.C.W. and Chow, W.K. (2019) 5D Model Theory for Creating of Life Forms. Journal of Modern Physics, 10, 1548-1565. https://doi.org/10.4236/jmp.2019.1013103

[8] Ching, W.Y., Xu, Y.N., Zhao, G.L., Wong, K.W. and Zandiehnadem, F. (1987) Electronic Structure and Excitonic-Enhanced Superconducting Mechanism in $\mathrm{YBa}_{2} \mathrm{Cu}_{3} \mathrm{O}_{7-\delta}$, Physical Review Letters, 59, 1333-1336. https://doi.org/10.1103/PhysRevLett.59.1333

[9] Wong, K.W. and Ching, C.W. (1989) The Theory of Simultaneous Excitonic-Superconductivity Condensation. Physica C, 158, 1-14.

https://doi.org/10.1016/0921-4534(89)90294-3 